\title{
ON COMMUTATIVE POWER-ASSOCIATIVE NILALGEBRAS OF LOW DIMENSION
}

\author{
MURRAY GERSTENHABER ${ }^{1}$ AND HYO CHUL MYUNG
}

ABSTRACT. Commutative power-associative nilalgebras of dimension 4 and characteristic $\neq 2$ are shown to be nilpotent and all their isomorphism classes are determined.

The long-standing conjecture, originally due to A. A. Albert, that a commutative power-associative nilalgebra of finite dimension over a field is nilpotent, has recently been disproved by Suttles [4], who gave a counterexample of dimension 5. This dimension is generally the best possible, for we show here that every commutative power-associative nilalgebra of dimension 4 over a field of characteristic $\neq 2$ is nilpotent, and we determine the isomorphism classes of all such algebras. The proof is elementary and cannot be significantly simplified by the use of the general results of [1] and [2], which require, more over, additional assumptions about the characteristic.

Throughout, $A$ will denote a commutative power-associative nilalgebra of dimension 4 over a field $F$ of characteristic $\neq 2$. The subspace of $A$ generated by elements $u, v, w, \cdots$ will be denoted by $(u, v, w, \cdots)$. Since every $x \in A$ is nilpotent, the powers of $x$ are linearly independent, so we must have $x^{n}=0$ for $n \geq 5$; the least $n$ such that $x^{n}=0$ for all $x \in A$ is called the nilindex. Now the product of any two elements of $A$ can be written as a linear combination of squares, for $x y=1 / 2\left[(x+y)^{2}-x^{2}-y^{2}\right]$. Therefore, if the nilindex is 2 , then every product vanishes. If it is 5 , then $A=\left(x, x^{2}, x^{3}, x^{4}\right)$ for any $x$ with $x^{4} \neq 0$. These are trivial cases, in each of which there is, up to isomorphism, a unique, associative, algebra. Only the cases of nilindex 3 and 4 are of interest.

1. Nilindex 3. Linearizing the identity $\left(x^{2}\right) x=0$ yields $2[(x y) z+(y z) x+(z x) y]=0$ for all $x, y, z \in A$. Therefore, denoting right

Received by the editors January 2, 1974.

AMS (MOS) subject classifications (1970). Primary 17 A10.

Key words and phrases. Nilalgebra.

1 The first author gratefully acknowledges the support of the NSF through a grant to the University of Penn sylvania. 
multiplication by $x$ by $R_{x}$ we have, for all $y, z \in A$,

$$
R_{y} R_{z}+R_{z} R_{y}=-R_{y z}
$$

Setting $y=z=x$ gives

$$
R_{x}^{2}=-2 R_{x^{2}}
$$

Setting $y=x, z=x^{2}$ in (1), and noting that $x^{3}=0$, gives $R_{x} R_{x^{2}}+R_{x}{ }^{2}{ }_{x}=$ 0 , which with (2) implies that $R_{x}^{3}=0$. Now choose any $x \in A$ with $x^{2} \neq 0$, and set $X=\left(x, x^{2}\right)$. This is carried into itse lf by $R_{x}$, which therefore operates on the two-dimensional quotient $A / X$. As $R_{x}$ is nilpotent, we have $R_{x}^{2}(A / X)=0$, so $(y x) x \in X$ for all $y \in A$, i.e., $(y x) x=\alpha x+\beta x^{2}$ for some $\alpha$, $\beta \in F$. Since $R_{x}^{3}=0$, multiplying by $x$ shows that $\alpha=0$, after which using (2) and the fact that $R_{y}$ is also nilpotent shows $\beta=0$ also. Thus $y x^{2}=0$, and since every product is a linear combination of squares, this shows that the product of any three elements of $A$ is zero. In particular, $A$ is associative, so we have

Theorem 1. A commutative power-associative nilalgebra A of nilindex 3 and of dimension 4 over a field $F$ of characteristic $\neq 2$ is associative, and $A^{3}=0$.

These algebras being associative, their classification is well known; cf. Kruse and Price [3, Chapter VI]: If $\operatorname{dim} A^{2}=1$, then one defines a symmetric bilinear form on the 3 -dimensional space $A / A^{2}$ by choosing any $x$ with $x^{2} \neq 0$ and defining the product of the cosets of $u, v \in A$ to be $\alpha$ whenever $u v=a x^{2}$; with respect to this form the length of $x$ itself is clearly 1. The problem of classifying these algebras up to isomorphism is identical with that of classifying such forms with the additional condition that there be a vector of length 1 . Unfortunately, this problem is completely solved only for certain special fields, e.g., the real and complex numbers. If $\operatorname{dim} A^{2}=2$, the only other possibility, then one chooses $x, y \in A$ such that $x^{2}$ and $x y$ span $A^{2}$. Subtracting, if necessary, a multiple of $x$ from $y$ one can, moreover, so choose $y$ such that $y^{2}=\alpha x^{2}$ for some $\alpha \in F$. It is easy to check that if one takes any other $x$ and $y$ with these properties, then $\alpha$ is replaced by $a c^{2}$ for some $c \neq 0$. Therefore, denoting the multiplicative group of $F$ by $F^{*}$, these algebras are parameterized by the elements of $F^{*} /\left(F^{*}\right)^{2}$ and 0 .

2. Nilindex 4. Choose any $x$ with $x^{3} \neq 0$ and set $X=\left(x, x^{2}, x^{3}\right)$. We claim $\left(x^{2}, x^{3}\right)=A^{2}$. It is sufficient to show that $y^{2} \in\left(x^{2}, x^{3}\right)$ for all $y$, and 
we may further suppose $y \notin X$ and $y^{2} \neq 0$, else the matter is trivial. Set $Y=$ $\left(y, y^{2}, y^{3}\right)$. Then $X \cap Y$ is a proper subalgebra of $X$, hence must be contained in $\left(x^{2}, x^{3}\right)$, and is a subalgebra of $Y$ of dimension equal to $\operatorname{dim} Y-1$ (since $\operatorname{dim} X=3$ ), and therefore must contain $y^{2}$; thus $y^{2} \in\left(x^{2}, x^{3}\right)$ as asserted. It follows that $A^{2} A^{2}=0$. Now $y$ being arbitrary, we have $y x^{2} \in A^{2}$, hence $y x^{2}=a x^{2}+b x^{3}$ for some $a, b \in F$. We claim $a=0$. Otherwise, setting $z=(1 / a)(y-b x)$, we have $z x^{2}=x^{2}$; computing $\left[\left(z+x^{2}\right)^{2}\left(z+x^{2}\right)\right]$ - $\left(z+x^{2}\right)$, which must vanish, we, find, using the fact that $A^{2} A^{2}=0$, that it is $2 x^{2}$, a contradiction. If now we replace $x$ by $x+x^{2}$, thereby replacing $x^{2}$ by $x^{2}+2 x^{3}$ but leaving $x^{3}$ unchanged, it follows that $y x^{3}$ is also a multiple of $x^{3}$. In fact, $y x^{3}=0$, for if $y x^{3}=d x^{3}$, then computing $\left[\left(y+x^{3}\right)^{2}\left(y+x^{3}\right)\right]$ - $\left(y+x^{3}\right)$, which must vanish, one gets $2 d^{3} x^{3}$, so $d=0$. We see now that replacing the original $y$ by $y-b x$, for which we have $(y-b x) x^{2}=0$, one can so choose $y$ such that $y \notin X$ and $y x^{2}=y x^{3}=0$, so $y A^{2}=0$. The product of any four of the elements $x, x^{2}, x^{3}, y$ vanishes, and as these $\operatorname{span} A$, it follows that the product of any 4 elements of $A$ vanishes, so $A^{4}=0$. Therefore, we have

Theorem 2. If $A$ is a commutative power-associative nilalgebra of nilindex 4 and of dimension 4 over a field $F$ of characteristic $\neq 2$, then $A^{4}=0$ and there is $y \notin A^{2}$ such that $y A^{2}=0$.

The $y$ of the theorem is not unique, but as $\operatorname{dim} A / A^{2}=2$, there cannot be, modulo $A^{2}$, two independent elements both annihilating $A^{2}$, so $y^{2}$ is determined up to multiplication by an element of $\left(F^{*}\right)^{2}$. As before, $x$ will denote an element of $A$ such that $x^{3} \neq 0$; then clearly $y \notin\left(x, x^{2}, x^{3}\right)$ so $A=$ $\left(x, x^{2}, x^{3}, y\right)$. We have the following possibilities:

1. We can so choose $x$ and $y$ such that $y x=0$. If $y^{2}=0$ then $A$ is unique, the direct sum of $(y)$ and $\left(x, x^{2}, x^{3}\right)$, and is associative. If $y^{2}=\beta x^{3}$ with $\beta \neq 0$, setting $y^{\prime}=y / \beta$ and $x^{\prime}=x / \beta$ gives $y^{\prime 2}=x^{\prime 3}$. This unique algebra is also associative. If $y^{2}=\alpha x^{2}+\beta x^{3}$ with $\alpha \neq 0$, replacing $x$ by $x+(\beta / 2 \alpha) x^{2}$ shows we may assume $\beta=0$. Clearly $\alpha$ is determined at most up to multiplication by an element of $\left(F^{*}\right)^{2}$, and it is easy to check that another choice of $x$ and $y$ replaces $\alpha$ by $\alpha c^{2}$ for some $c \neq 0$, so we have a family of algebras, all nonassociative, parameterized by the elements of $F^{*} /\left(F^{*}\right)^{2}$.

2. We cannot so choose $x$ and $y$ such that $y x=0$. Therefore we cannot have $y x=\beta x^{3}$ since $x\left(y-\beta x^{2}\right)=0$. Choosing any $x$ with $x^{3} \neq 0$, we may assume that $y x=\gamma x^{2}+\delta x^{3}$ with $y \neq 0$. Replacing $x$ by $\gamma x+(\delta / 2) x^{2}$ shows we 
may so choose $x$ such that $y x=x^{2}$. If $y^{2}=0$ we have a unique nonassociative algebra. If $y^{2}=\beta x^{3}$ with $\beta \neq 0$, replacing $x$ by $x / \beta$ and $y$ by $y / \beta$ shows we may assume $\beta=1$ and we have a unique algebra, which is not associative. Finally, if $y^{2}=\alpha x^{2}+\beta x^{3}$ with $\alpha \neq 0$, then as $y(y-\alpha x)=\beta x^{3}$, we must have $(y-\alpha x)^{3}=0$ (else we would replace $x$ by $y-\alpha x$ ); the left side is $\alpha^{2}(1-\alpha) x^{3}$, so $\alpha=1$. Replacing $x$ by $x+(\beta / 2) x^{2}$ and $y$ by $y+\beta x^{2}$, shows we may assume $\beta=0$, so we have a unique algebra given by $y^{2}=$ $y x=x^{2}$ and, as always, $y x^{2}=y x^{3}=0$. It is not associative. This ends the classification. We have found one family of algebras parameterized by $F^{*} /\left(F^{*}\right)^{2}$, and 5 individual algebras of which precisely 2 are associative.

\section{REFERENCES}

1. A. A. Albert, Power-associative rings, Trans. Amer. Math. Soc. 64 (1948), 552-593. MR 10, 349 .

2. M. Gerstenhaber, On nilalgebras and linear varieties of nilpotent matrices. II, Duke Math. J. 27 (1960), 21-31. MR 22 \#4742.

3. R. L. Kruse and D. T. Price, Nilpotent rings, Gordon and Breach, New York, 1969. MR $42 \# 1858$.

4. D. Suttles, A counterexample to a conjecture of Albert, Notices Amer. Math. Soc. 19 (1972), A-566. Abstract \#72T-A169.

DE PARTMENT OF MATHEMATICS, UNIVERSITY OF PENNSYLVANIA, PHILADELPHIA, PENNSYLVANIA 19174

DEPARTMENT OF MATHEMATICS, UNIVERSITY OF NORTHERN IOWA, CEDAR FALLS, IOWA 50613 